\begin{tabular}{|c|c|}
\hline Title & Microstructural A Iterations in Bipolar and Major Depressive Disorders: A Diffusion Kurtosis Imaging Study \\
\hline Author(s) & $\begin{array}{l}\text { Sawamura, Dai suke; Narita, Hisashi; Hashimoto, Naoki; Nakagawa, Shin; Hamaguchi, Hiroyuki; Fujima, Noriyuki; } \\
\text { Kudo, Kohsuke; Shirato, Hiroki; Tha, Khin K. }\end{array}$ \\
\hline Citation & $\begin{array}{l}\text { Journal of magnetic resonance imaging, 52(4), 1187-1196 } \\
\text { https://doi.org/10.1002/Jmri.27174 }\end{array}$ \\
\hline Issue Date & $2020-10$ \\
\hline Doc URL & http:/hdl.handle.net/2115/82885 \\
\hline Rights & $\begin{array}{l}\text { This is the peer reviewed version of the following article: Sawamura, D., Narita, H., Hashimoto, N., Nakagawa, S., } \\
\text { Hamaguchi, H., Fujima, N., Kudo, K., Shirato, H. and Tha, K.K. (2020), Microstructural A Iterations in Bipolar and } \\
\text { Maj or Depressive Disorders: A Diffusion Kurtosis Imaging Study. J Magn Reson Imaging, 52: 1187-1196. } \\
\text { doi:10.1002/mri.27174, which has been published in final form at https://doi.org/10.1002/jmri.27174. This article may } \\
\text { be used for non-commercial purposes in accordance with Wiley Terms and Conditions for U se of Self-A rchived } \\
\text { V ersions. }\end{array}$ \\
\hline Type & article (author version) \\
\hline Additional Information & There are other files related to this item in HUSCAP. Check the above URL. \\
\hline File Information & J Magn Reson Imaging_52_1187.pdf \\
\hline
\end{tabular}

Instructions for use 


\section{Microstructural Alterations in Bipolar and Major Depressive Disorders: A \\ Diffusion Kurtosis Imaging Study}

Daisuke Sawamura, $\mathrm{PhD}^{1,2}$, Hisashi Narita, $\mathrm{MD}, \mathrm{PhD}^{3}$, Naoki Hashimoto, $\mathrm{MD}, \mathrm{PhD}^{3}$, Shin Nakagawa, MD, $\mathrm{PhD}^{3}$, Hiroyuki Hamaguchi, BS, $\mathrm{MSc}^{4}$, Noriyuki Fujima, MD, $\mathrm{PhD}^{5,6}$, Kohsuke Kudo, MD, $\mathrm{PhD}^{5,7}$, Hiroki Shirato, $\mathrm{MD}, \mathrm{PhD}^{7}$, Khin K. Tha, MD, $\mathrm{PhD}^{1,5,7,8}$ Department of Biomarker Imaging Science, Hokkaido University Graduate School of Medical Science and Engineering ${ }^{1}$; Department of Functioning and Disability, Hokkaido University Faculty of Health Sciences²; Department of Psychiatry, Hokkaido University Graduate School of Medicine ${ }^{3}$; Departments of Radiological Technology ${ }^{4}$ and Diagnostic and Interventional Radiology ${ }^{5}$, Hokkaido University Hospital; Departments of Radiology, Otolaryngology-Head and Neck Surgery, and Radiation Oncology, Boston Medical Center, Boston University School of Medicine ${ }^{6}$; Global Station for Quantum Medical Science and Engineering, Global Institution for Collaborative Research and Education, Hokkaido University'; Global Center for Biomedical Science and Engineering, Hokkaido University Faculty of Medicine ${ }^{8}$

\section{Corresponding Author}

Khin Khin Tha,

Global Center for Biomedical Science and Engineering,

Hokkaido University Faculty of Medicine, 
N15, W7, Kita-ku, Sapporo,

060-8638, Japan

Email: kktha@med.hokudai.ac.jp

Phone: 011-706-8183

\section{Grant Support}

This study was partly supported by (1) the Global Station for Quantum Medical Science, Global Institution for Collaborative Research and Education, Hokkaido University and (2) the Grantin-Aid for Scientific Research by the Japan Society for Promotion of Science (KAKENHI Grant Number 18H06081).

\section{Running Title}

DKI Alterations in BD and MDD 


\title{
Microstructural Alterations in Bipolar and Major Depressive Disorders: A Diffusion Kurtosis Imaging Study
}

\begin{abstract}
BACKGROUND: Identifying structural and functional abnormalities in bipolar (BD) and major depressive disorders (MDD) is important for understanding biological processes.

HYPOTHESIS: Diffusion kurtosis imaging (DKI) may be able to detect the brain's microstructural alterations in BD and MDD and any differences between the two.

STUDY TYPE: Prospective
\end{abstract}

SUBJECTS: 16 BD patients, 19 MDD patients, and 20 age-and gender-matched healthy volunteers.

FIELD STRENGTH/SEQUENCE: DKI at 3.0T

ASSESSMENT: The major DKI indices of the brain were compared voxel-by-voxel among the three groups. Significantly different voxels were tested for correlation with clinical variables \{i.e., Young Mania Rating Scale (YMRS), 17-item Hamilton Depression Rating Scale (17-HDRS), Montgomery-Åsberg Depression Rating Scale (MADRS), total disease duration, duration of current episode, and the number of past manic/ depressive episodes\}. The performance of the DKI indices in identifying microstructural alterations was estimated.

STATISTICAL TESTS: One-way ANOVA was used for group comparison of DKI indices. The performance of these indices in detecting microstructural alterations was determined by ROC analysis. Pearson's product-moment correlation analyses were used to test the correlations of these indices with clinical variables.

RESULTS: DKI revealed widespread microstructural alterations across the brain in each disorder $(\mathrm{P}<0.05)$. Some were significantly different between the two disorders. Mean kurtosis (MK) in the gray matter of the right inferior parietal lobe was able to distinguish BD and MDD 
with an accuracy of 0.906 . There revealed a strong correlation between MK in that region and YMRS in BD patients $(\mathrm{r}=-0.641$, corrected $\mathrm{P}=0.042)$ or $17-\mathrm{HDRS}$ in MDD patients $(\mathrm{r}=-$ 0.613, corrected $\mathrm{P}=0.030)$. There were also strong correlations between a few other DKI indices and disease duration $(\mathrm{r}=-0.676$ or 0.626 , corrected $\mathrm{P}<0.05)$.

DATA CONCLUSION: DKI detected microstructural brain alterations in BD and MDD. Its indices may be useful to distinguish the two disorders or to reflect disease severity and duration. 
DKI Alterations in BD and MDD 3

\section{KEYWORDS}

Diffusion kurtosis imaging

Mean kurtosis

Bipolar disorder

Major depressive disorder

Microstructural alterations 


\section{INTRODUCTION}

Bipolar disorder (BD) and major depressive disorder (MDD) are the two most frequent mood disorders. The lifetime prevalence of these two disorders together approximate $20.0 \%$ (1). BD is characterized by recurrent episodes of elevated mood and depression, whereas MDD persistent low mood, loss of interest, fatigue, and low energy (2). Distinguishing these two disorders can sometimes be challenging since $\mathrm{BD}$ patients often begin with a depressive episode and remain depressive most of the time during the disease course (3). There has been evidence that BD is often misdiagnosed as MDD (3). Since the treatment regime is different between these two disorders, correct early diagnosis is essential.

Recent evidence suggests differences in biological processes between these two disorders. First, gene expression profiles tend to vary. MDD has been reported as associated with five polymorphisms within the FKBP5 gene and to have shorter hippocampal telomere length $(4,5)$. On the other hand, lower relative expression levels of brain-derived neurotrophic factor (BDNF) mRNA have been detected in the peripheral venous blood samples of BD patients than MDD patients (6). Second, biochemical profiles also tend to vary. A positive correlation between the plasma cortisol level and serotonin transporter availability has been observed in the caudate nucleus and midbrain in MDD, but not in $\mathrm{BD}$ (7). Lower concentrations of choline and creatine compounds and $\mathrm{N}$-acetyl aspartate have been reported in the parietal cortex of BD patients and posterior cingulate cortex of MDD patients (8). Third, the findings of neuroimaging studies also suggest differences in cortical thickness, functional connectivity, and cerebral blood flow in the frontal, parietal, and temporal lobes, between these two disorders (9-12). Three existing diffusion kurtosis imaging (DKI) studies also report lower axial kurtosis (AK) in the right putamen and higher mean diffusivity (MD) in the right caudate nucleus and superior cerebellar peduncle of BD patients than their MDD counterpart (11-13). There have also been diffusion tensor imaging (DTI) studies that report lower fractional anisotropy (FA) 
in the anterior cingulate, corpus callosum, uncinate fasciculus, corticospinal tract, and superior longitudinal fasciculus in BD than MDD (14-18). However, reliable conclusions are yet to be made due to the small number of studies and inconsistent findings among them.

MR imaging offers in vivo and noninvasive visualization of biological processes. Of several techniques, DKI is known to detect microstructural gray and white matter abnormalities through quantification in the deviation of water molecule diffusion from Gaussianity $(19,20)$. DKI has been widely accepted as more accurate and robust than DTI (21). It's another advantage, compared to the other advanced diffusion imaging sequences, is the easiness to perform. Its performance has been satisfactory and superior to DTI in identifying microstructural abnormalities in several neurodegenerative diseases and psychiatric disorders, including BD and MDD (22-25). As mentioned previously, the observations of the initial DKI studies are suggestive of microstructural differences between $\operatorname{BD}$ and $\operatorname{MDD}(11,12)$. Nevertheless, the existing DKI studies that attempt to identify or distinguish the microstructural alterations in BD and MDD are limited in number and fail to cover the whole brain (i.e., the evaluation was limited to the gray matter nuclei and part of the cerebellum) (11-13). From the previous DTI and DKI reports of BD and MDD, these disorders can have different DKI characteristics in those areas left unexplored $(24,25)$. Moreover, these characteristics may have clinical importance in distinguishing these two disorders or may serve as imaging indicators of disease severity.

This prospective study was aimed to evaluate the microstructural alterations of the brain in BD and MDD by using the whole brain voxel-based DKI analysis.

\section{MATERIALS AND METHODS}

This prospective study was approved by the local institutional Ethical Review Board. Written informed consent was obtained from all participants. 


\section{Participants}

The patients were recruited during a 60-month period (October 2012 to October 2017).

The inclusion criteria were age between 20 and 64 years and the diagnosis of BD or MDD based on the Structured Clinical Interview for Axis I Disorders according to the Diagnostic and Statistical Manual, Fourth Edition, Text Revision (DSM-IV-TR) (26). The exclusion criteria were an absolute contraindication to MR imaging, comorbidity of axis I or II disorders, history of uncontrolled cerebrovascular diseases, substance abuse or dependency, and any abnormality on conventional MR imaging sequences \{i.e., T2-weighted imaging (T2WI) and fluidattenuated inversion recovery (FLAIR) imaging sequences\}, or visible artifacts on the DKI and three-dimensional magnetization-prepared rapid acquisition gradient-echo imaging (MPRAGE) datasets as evaluated by a neuroradiologist (19-year experience). Seventeen BD patients and 19 MDD patients enrolled in the study\{The sample size required to distinguish BD and MDD by FA of DTI is 16 for each group if the probabilities of type I and type II errors are set as 0.05 (18) . All patients were receiving psychotropic medications $(15,17,18)$.

We also recruited twenty age-, gender-, and years of education-matched healthy volunteers in this study. The additional exclusion criterion for these volunteers was the history of psychiatric illnesses, which extended to first-degree relatives.

\section{Assessment of Psychiatric Symptoms}

All patients were evaluated for psychiatric symptoms at the time of initial hospital consultation, using the following clinical scales: Young Mania Rating Scale (YMRS), 17-item Hamilton Depression Rating Scale (17-HDRS), and Montgomery-Åsberg Depression Rating Scale (MADRS) (27-29). Total disease duration, the duration of the current disease episode, the number of past manic/depressive episodes, and the history of medication were also noted. 
All assessments were performed by a board-certified psychiatrist.

\section{MR Imaging}

MR imaging was acquired using a 3.0 Tesla scanner (Achieva TX; Philips Medical Solutions, Best, the Netherlands) and a standard 32-channel head coil. MR imaging was performed within two weeks from the symptom assessment. Padding and fixation device were used to minimize head motion during acquisition. DKI was acquired with the following scan parameters: repetition time $(\mathrm{TR}) /$ echo time $(\mathrm{TE})=5032 / 85 \mathrm{~ms}$, flip angle $=90^{\circ}$, voxel size $=2.9$ $\times 3.1 \times 3.0 \mathrm{~mm}^{3}$, number of excitation $(\mathrm{NEX})=1$, gapless, 43 axial sessions, $b=0,1000$, and $2000 \mathrm{~s} / \mathrm{mm}^{2}$, 32 diffusion gradient directions, and scan time= 7:54 min. Three-dimensional MPRAGE was also acquired for construction of brain mask and evaluation of any structural changes $\left(\mathrm{TR} / \mathrm{TE}=6.8 / 3.1 \mathrm{~ms}\right.$, inversion time $(\mathrm{TI})=1100 \mathrm{~ms}$, flip angle $=8^{\circ}$, voxel size $=1 \times 1$ $\times 1.2 \mathrm{~mm}^{3}, \mathrm{NEX}=1$, gapless, 170 sagittal sessions $)$. Axial T2WI $(\mathrm{TR} / \mathrm{TE}=4137 / 90 \mathrm{~ms})$ and FLAIR imaging $(\mathrm{TR} / \mathrm{TE}=10000 / 100 \mathrm{~ms}, \mathrm{TI}=2700 \mathrm{~ms})$ were also acquired to exclude gross abnormalities. Visual review of MR imaging data was performed by an author with 19 years of experience in neuroimaging. The DKI and MPRAGE images with visible artifacts were excluded from further analysis, which resulted in the exclusion of one BD patient.

\section{Image Preprocessing}

Image realignment and correction for eddy current distortion were performed at the MR imaging operator console. Diffusional Kurtosis Estimator (DKE) version 2.6.0 (MUSC Center for Biomedical Imaging, Charleston, USA) was used to generate mean kurtosis (MK), AK, radial kurtosis (RK), MD, axial diffusivity (AD), radial diffusivity (RD), FA, and kurtosis fractional anisotropy (KFA) maps from the DKI data (19). The MK maps of all participants were then spatially normalized by using the default parameters of SPM12 (Wellcome Centre 
for Human Neuroimaging, London, UK), which runs on MATLAB R2010b (Mathworks, Natick, USA). Next, the mean normalized MK map was generated and smoothed with a $6 \mathrm{~mm}$ full width half maximum (FWHM) Gaussian kernel to form a customized MK tissue probability map. We then repeated the spatial normalization of MK maps using the default parameters of SPM12 but with the customized MK tissue probability map this time (30). The transformation parameters were applied to all other index maps of DKI. All spatially normalized DKI maps were then smoothed with a 6-mm FWHM Gaussian kernel.

Spatial normalization of all MPRAGE images and subsequent segmentation of the gray and white matter were performed by using the default parameters of SPM12. A brain mask that included only the brain parenchyma (i.e., the gray and white matter) of the participants was generated. The segmented gray and white matter were then smoothed with a 2-mm FWHM Gaussian kernel, to compare their densities.

\section{Data Analysis}

All statistical analyses were performed using SPM12 or the Statistical Package for the Social Sciences (SPSS) 24.0 for Windows (Chicago, Illinois).

\section{Demographic and Clinical Variables}

Age, gender, and years of education were compared among the three groups by using a one-way analysis of variance (ANOVA) or chi-square $\left(\chi^{2}\right)$ tests. The clinical scales, disease duration, and the number of past manic/depressive episodes were compared between BD and MDD by using Student's t- and $\chi^{2}$ tests. For all comparisons, statistical significance was set as $\mathrm{P}<0.05$ after correction for multiple comparisons.

\section{Group Differences in DKI Alterations}


The major DKI indices of the brain parenchyma covered by brain mask were compared voxel-by-voxel among the three groups by using one-way ANOVA. $\mathrm{P}<0.001$ for clusters containing at least 50 voxels was considered statistically significant. Age, gender, and years of education were considered as covariates. The mean values of significant clusters, if any, were extracted by using MarsBar-0.44 (The fMRI Centre of Marseilles, Marseille Cedex, France) and compared among the groups by using Bonferroni post hoc tests. The WFU PickAtlas version 2.4 (Wake Forest University Health Sciences, North Carolina, USA) was used to locate each brain region.

To evaluate if the microstructural alterations detected by DKI were accompanied by structural changes, the gray and white matter densities of the brain were compared among the three groups by using one-way ANOVA and Bonferroni post hoc tests. As in DKI data analysis, $\mathrm{P}<0.001$ for clusters containing at least 50 voxels was considered significant, and age, gender, and years of education were considered as covariates.

\section{Effect of Psychotropic Medications on the DKI Alterations}

For each patient group, the mean values of significant DKI clusters revealed by oneway ANOVA were compared between those receiving a specific psychotropic medication (i.e., antidepressant, mood stabilizer, or antipsychotic drug) and those did not. Student's t-tests were used, and statistical significance was set as $\mathrm{P}<0.05$.

\section{Clinical Relationships of the DKI Alterations}

The mean values of the clusters with significant DKI alterations in the patients were then tested for correlation with the clinical variables (i.e., total disease duration, duration of current episode, number of past manic/ depressive episodes, and the clinical scales) by using Pearson's product-moment correlation analyses, controlling for covariates. The P values were 
corrected for the number of clinical variables evaluated, and corrected $\mathrm{P}<0.05$ was considered significant. The diagnostic performance of DKI indices in distinguishing the three groups was determined by using receiver operating characteristic (ROC) analysis. Sensitivity and specificity were defined as the maximum on each ROC curve. The best cut-off values to distinguish the three groups were also determined. The area under curves (AUC) and 95\% confidence intervals were estimated through bootstrapping with 1000 repetitions (31).

\section{RESULTS}

\section{Demographic and Clinical Variables}

The demographic and clinical variables are summarized in Table 1 . There were no significant differences in age, gender, and years of education among the three groups. The BD patients tended to have longer total disease duration than MDD patients. The duration of the current disease episode was significantly longer in MDD. The BD patients had a larger number of past manic/depressive episodes than MDD patients. The two groups did not differ significantly in the severity of clinical symptoms. One BD and two MDD patients were in complete remission. Fourteen BD and 17 MDD patients were in the depressive episode. A BD patient was in the mixed episode. No patient was in the manic episode (27-29).

\section{Group Differences in DKI Alterations}

Fig 1s shows the results of the voxel-by-voxel comparison of the major DKI indices among the three groups, by using one-way ANOVA. There were several significant clusters, and their numbers were five each for MK and AK, four for RK, 10 for MD, eight for AD, 11 for RD, seven for FA, and 15 for KFA.

Of these clusters, post hoc analyses revealed that the MDD patients had significantly higher MK in the gray matter of the right inferior parietal lobe, higher MK and RK in the white 
matter of the right precentral gyrus, higher AK in the white matter of the right supramarginal gyrus, left putamen, and the left side of the midbrain, lower MD, AD and RD in the gray matter of the right precuneus and bilateral cunei, lower $\mathrm{RD}$ in the right culmen, higher KFA in the white matter of the right superior frontal gyrus and left temporal lobe, and lower KFA in the white matter of the right cingulate gyrus and right inferior frontal operculum, than the BD patients (Fig 1). Of note, MK in the gray matter of the right inferior parietal lobe and AK in the white matter of the right supramarginal gyrus of the MDD patients and MK and RK in the white matter of the right precentral gyrus of both patient groups were higher than those of the healthy volunteers.

In addition to these differences between the two patient groups, the post hoc analyses also revealed significant alterations in one or more of the DKI indices — i.e., lower mean or directional kurtosis, higher or lower mean or directional diffusivity, and lower anisotropy — in widespread brain regions including bilateral frontal, parietal, temporal, and limbic lobes, left putamen, right thalamus, and brainstem, in both patient groups compared to healthy volunteers. The lesion distributions are detailed in Table 1s. The MDD patients additionally had lower MD, $\mathrm{AD}$ and $\mathrm{RD}$ in the gray matter of the right precuneus and bilateral cunei, lower $\mathrm{RD}$ in the right culmen, and lower KFA in the right temporal white matter than the healthy volunteers.

No significant difference in the gray or white matter density was observed among the three groups.

\section{Effect of Psychotropic Medications on the DKI Alterations}

Of 65 clusters given in Fig 1s and Table 1s, the mean values of 11 clusters (i.e., five, two, and four clusters for an antidepressant, mood stabilizer, and antipsychotic drug, respectively) differed between the $\mathrm{BD}$ patients receiving medications and those who did not. For MDD patients, there was only one cluster which differed significantly between those 
receiving mood stabilizer and those who did not. The mean values of these clusters are given in Fig 2s.

\section{Clinical Relationships of the DKI Alterations}

A significant strong negative correlation was observed between MK in the gray matter of the right inferior parietal lobe and YMRS in the BD patients $(r=-0.641$, corrected $\mathrm{P}=0.042)$ (Fig 2a) or 17-HDRS in the MDD patients $(r=-0.613$, corrected $\mathrm{P}=0.030$ ) (Fig 2b). KFA of the right inferior frontal operculum $(\mathrm{r}=-0.676$, corrected $\mathrm{P}=0.012$ ) (Fig 3a) and $\mathrm{MD}$ in the white matter of the right orbital gyrus ( $r=0.626$, corrected $\mathrm{P}=0.024$ ) (Fig 3b) showed significant strong negative and positive correlations with total disease duration, in the MDD patients. MD in the white matter of triangular part of the right inferior frontal gyrus showed a significant strong positive correlation with the duration of current disease episode in the BD patients $(r=0.696$, corrected $\mathrm{P}=0.018)($ Fig 3c).

Table 2 summarizes the results of the ROC analysis. The best cut-off value was achieved with MK in the gray matter of the right inferior parietal lobe, which was 0.836 . With this value, the BD and MDD patients could be distinguished with sensitivity and specificity of 0.895 and 0.875 , respectively $(\mathrm{AUC}=0.906,95 \%$ confidence interval $=0.803-1.000$ ).

\section{DISCUSSION}

We report DKI comparisons of microstructural alterations throughout the brain between BD and MDD. The results suggest specific differences in microstructural organization of cerebral cortex and white matter between the two disorders, in addition to those in deep gray matter nuclei and cerebellar hemispheres $(11,12)$. Of these differences, MK in the gray matter of the right inferior parietal lobe might have diagnostic value in distinguishing BD and MDD. In this study, MK in this region declined in the order of MDD patients, healthy volunteers, and 
BD patients. The right inferior parietal cortex is known to involve in motor learning, execution, inhibition, cognition of time and space, reasoning, working memory, and attention (32). Higher local MK in MDD may be due to delayed or failed dendritic pruning probably associated with hypergyrification, cortical thickening, or both, whereas lower MK in BD may be the result of a decrease in the number of neurons or dendritic arborization (9, 33-36). Hypergyrification and cortical thickening of the right inferior parietal cortex have been reported in MDD, and focal cortical thinning has been documented in $\operatorname{BD}(9,33,34,36)$. Lack of significant difference in the gray and white matter densities may suggest that these microstructural alterations appear earlier or persist later than the structural abnormalities. The observation of similar pattern of AK alterations in the white matter of the right supramarginal gyrus — a part of the right inferior parietal lobe, may be viewed as reorganization of axons underlying the cortex with hypergyrification in MDD and axonal loss or degeneration in BDD (37). Similarly, lower MD, $\mathrm{AD}$, and $\mathrm{RD}$ values observed in the other cortical gray matter regions such as precuneus and cuneus and the right culmen in the MDD patients are also thought to support the consideration that MDD is characterized by delayed or failed dendritic pruning probably associated with hypergyrification or cortical thickening.

The observation of higher MK and RK in BD and MDD in the white matter of right precentral gyrus may suggest that similar microstructural alterations as those described above, i.e., reorganization in the white matter microstructure, encounter. A change in RK instead of AK is thought to indicate that a more pronounced alteration occurs in the direction perpendicular to the principal axis of white matter axons. An increase in oligodendrocyte density or cell clustering of astrocytes may be responsible (38). Antidepressant medication may have implicated in BD patients (39). Dysfunction in the dopamine pathways connecting the precentral gyrus and the midbrain or thalamus has been linked to psychomotor retardation in affective disorders (33). The observation of significant differences in MK and RK between BD 
and MDD may merely be a variation in the magnitude of alterations. However, the lack of significant overlying cortical changes may need further investigation. Altered DKI indices such as lower AK in the midbrain and putamen may also be related to dysfunction of the dopamine pathways.

The results of this study also show the widespread involvement of the brain microstructure in BD and MDD. Psychotropic medication may have affected a few clusters, but the majority of the observations are likely independent of the medication. The clusters which reveal significant alterations in the DKI indices in this study are known to play a role in mood regulation, and abnormalities in these regions have been reported in affective disorders (5, 8-12, 15-18, 40). The results of this study are thus thought to support the existing evidence about neural underpinnings associated with these affective disorders. The ability to distinguish BD and MDD from the healthy volunteers, with high accuracies, by KFA in the white matter of the right superior frontal gyrus and AK in the white matter of cingulate gyrus is thought to suggest the potential application of these indices in unveiling the state-dependent changes in tissue microstructure. The observation of the significant correlation of KFA in the right inferior frontal operculum and MD in the white matter of the right orbital gyrus and triangular part of the right inferior frontal gyrus with disease duration may imply that these regions are susceptible to early injury or less responsive to medication. The fewer number of significant clusters in BD patients may be due to a relatively larger proportion of these patients with mild depression.

\section{Limitations}

First, the duration of the current disease episode and the number of past manic/depressive episodes were not identical between BD and MDD. These differences may have confounded the results, but it is thought that the effect is negligible in most regions since 
there was a lack of significant correlation between these variables and DKI alterations in most regions of the brain. Second, the diagnostic accuracy was tested on the same sample. Confirmation with a separate validation dataset would be ideal, but bootstrapping was done to achieve robust results as possible. Third, the sample size is small. The results of this study need to be confirmed with studies involving larger sample sizes that include medication-naïve patients. Fourth, because this study was designed to evaluate if the major DKI indices could identify the microstructural alterations of the brain in BD and MDD, the reversibility of these lesions cannot be determined. A longitudinal study needs to be conducted to understand this. However, from the observation of high MK values in the gray matter of the right inferior parietal lobe in the MDD patients with complete remission, it is thought that the reversibility of the lesions does not occur at least immediately.

\section{Conclusion}

This study highlights the variation in microstructural alterations of the brain between $\mathrm{BD}$ and MDD, which may have potential diagnostic value in distinguishing the two disorders or potential clinical value by reflecting the disease severity. The observation about these variations may also be helpful in better understanding of the pathological processes occurring in these disorders and for future drug discovery. 


\section{REFERENCES}

1. Kessler RC, Berglund P, Demler O, Jin R, Merikangas KR, Walters EE. Lifetime prevalence and age-of-onset distributions of DSM-IV disorders in the National Comorbidity Survey Replication. Arch Gen Psychiatry 2005; 62: 593-602.

2. Depression WHO 2012. https://www.who.int/mental_health/management/depression/who_paper_depression_ wfmh_2012.pdf

3. Ghaemi SN, Ko JY, Goodwin FK. "Cade's disease" and beyond: misdiagnosis, antidepressant use, and a proposed definition for bipolar spectrum disorder. Can J Psychiatr 2002; 47: 125.

4. Szczepankiewicz A, Leszczyńska-Rodziewicz A, Pawlak J, et al. FKBP5 polymorphism is associated with major depression but not with bipolar disorder. $\mathrm{J}$ Affect Disord 2014; 164: 33-37.

5. Mamdani F, Rollins B, Morgan L, et al. Variable telomere length across post-mortem human brain regions and specific reduction in the hippocampus of major depressive disorder. Transl Psychiatry 2015; 15:5:e636.

6. Li Z, Zhang C, Fan J, et al. Brain-derived neurotrophic factor levels and bipolar disorder in patients in their first depressive episode: 3-year prospective longitudinal study. $\mathrm{Br} \mathrm{J}$ Psychiatry 2014; 205: 29-35.

7. Chou YH, Lirng JF, Hsieh WC, Chiu YC, Tu YA, Wang SJ. Neither cortisol nor brainderived neurotrophic factor is associated with serotonin transporter in bipolar disorder. Eur Neuropsychopharmacol 2016; 26: 280-287.

8. Li H, Xu H, Zhang Y, et al. Differential neurometabolite alterations in brains of medication-free individuals with bipolar disorder and those with unipolar depression: a two-dimensional proton magnetic resonance spectroscopy study. Bipolar Disord 
2016;18: 583-590.

9. Rubin-Falcone H, Zanderigo F, Thapa-Chhetry B, et al. Pattern recognition of magnetic resonance imaging-based gray matter volume measurements classifies bipolar disorder and major depressive disorder. J Affect Disord 2018; 227: 498-505.

10. Pang Y, Chen H, Wang Y, et al. Transdiagnostic and diagnosis-specific dynamic functional connectivity anchored in the right anterior insula in major depressive disorder and bipolar depression. Prog Neuropsychopharmacol Biol Psychiatry 2018; 85: 7-15.

11. Zhao L, Wang Y, Jia Y, et al. Cerebellar microstructural abnormalities in bipolar depression and unipolar depression: A diffusion kurtosis and perfusion imaging study. J Affect Disord 2016; 195: 21-31.

12. Zhao L, Wang Y, Jia Y, et al. Microstructural Abnormalities of Basal Ganglia and Thalamus in Bipolar and Unipolar Disorders: A Diffusion Kurtosis and Perfusion Imaging Study. Psychiatry Investig 2017; 14: 471-482.

13. Zhao L, Chen G, Jia Y, et al. Alteration of Red Nucleus Microstructure in Depressive Bipolar II Disorder and Unipolar Depression: A Diffusion Kurtosis and Perfusion Imaging Study. Neuropsychiatry-Lond 2019; 9: 2086-2097.

14. Matsuoka K, Yasuno F, Kishimoto T, et al. Microstructural Differences in the Corpus Callosum in Patients With Bipolar Disorder and Major Depressive Disorder. J Clin Psychiatry 2017; 78 :99-104.

15. Benedetti F, Absinta M, Rocca MA, et al. Tract-specific white matter structural disruption in patients with bipolar disorder. Bipolar Disord 2011; 13: 414-24.

16. Deng F, Wang Y, Huang H, et al. Abnormal segments of right uncinate fasciculus and left anterior thalamic radiation in major and bipolar depression. Prog Neuropsychopharmacol Biol Psychiatry. 2018; 81:340-349. 
17. Repple J, Meinert S, Grotegerd D, et al. A voxel-based diffusion tensor imaging study in unipolar and bipolar depression. Bipolar Disord 2017; 19: 23-31.

18. Versace A, Almeida JR, Quevedo K, et al. Right orbitofrontal corticolimbic and left corticocortical white matter connectivity differentiate bipolar and unipolar depression. Biol Psychiatry 2010; 68: 560-567.

19. Jensen JH, Helpern JA. MRI quantification of non-Gaussian water diffusion by kurtosis analysis. NMR Biomed 2010; 23: 698-710.

20. Tabesh A, Jensen JH, Ardekani BA, Helpern JA. Estimation of tensors and tensorderived measures in diffusional kurtosis imaging. Magn Reson Med 2011; 65: 823-836.

21. Cheung MM, Hui ES, Chan KC, Helpern JA, Qi L, Wu EX. Does diffusion kurtosis imaging lead to better neural tissue characterization? A rodent brain maturation study. NeuroImage 2009; 45: 386-392.

22. Kazumata K, Tha KK, Narita H, et al. Characteristics of Diffusional Kurtosis in Chronic Ischemia of Adult Moyamoya Disease: Comparing Diffusional Kurtosis and Diffusion Tensor Imaging. AJNR Am J Neuroradiol 2016; 37: 1432-1439.

23. Narita H, Tha KK, Hashimoto N, et al. Mean kurtosis alterations of cerebral white matter in patients with schizophrenia revealed by diffusion kurtosis imaging. Prog Neuropsychopharmacol Biol Psychiatry 2016; 71:169-75.

24. Ota M, Noda T, Sato N, et al. The use of diffusional kurtosis imaging and neurite orientation dispersion and density imaging of the brain in major depressive disorder. $\mathrm{J}$ Psychiatr Res 2018; 98: 22-29.

25. Ota M, Noda T, Sato N, et al. The use of diffusional kurtosis imaging and neurite orientation dispersion and density imaging of the brain in bipolar disorder. J Affect Disord 2019; 251:231-234.

26. First MB, Sptzer RL, Gibbon M, Williams JBW. Structured Clinical Interview for DSM-IV Axis I Disorders (SCID-I/P, Version 2.0). Biometrics Research Dept., New 
York State Psychiatric Institute; New York: 1995.

27. Tohen MI, Calabrese JR, Sachs GS, et al. Randomized, placebo-controlled trial of olanzapine as maintenance therapy in patients with bipolar I disorder responding to acute treatment with olanzapine. Am J Psychiatry 2006; 163: 247-256.

28. Chengappa KN, Hennen J, Baldessarini RJ, et al. Recovery and functional outcome following olanzapine treatment for bipolar I mania. Bipolar Disord 2005; 7: 68-76.

29. Carmody TJ, Rush AJ, Bernstein J, et al. The Montgomery Asberg and the Hamilton ratings of depression: a comparison of measures. Eur Neuropsychopharmacol 2006; 16: 601-611.

30. Ahn SJ, Kyeong S, Suh SH, Kim JJ, Chung TS, Seok JH. What is the impact of child abuse on gray matter abnormalities in individuals with major depressive disorder: a case control study. BMC Psychiatry 2016; 16: 397.

31. Skalska H, Freylich V. Web-Bootstrap Estimate of Area Under ROC Curve. Austrian J Stat. 2006; 35: 325-330.

32. Luo L, Wu K, Lu Y, et al. Increased Functional Connectivity Between Medulla and Inferior Parietal Cortex in Medication-Free Major Depressive Disorder. Front Neurosci 2018; 12: 926.

33. Peng D, Shi F, Li G, et al. Surface vulnerability of cerebral cortex to major depressive disorder. PLoS One. 2015; 10: e0120704.

34. Qiu L, Lui S, Kuang W, et al. Regional increases of cortical thickness in untreated, firstepisode major depressive disorder. Transl Psychiatry 2014; 4:e378.

35. Genç E, Fraenz C, Schlüter C, et al Diffusion markers of dendritic density and arborization in gray matter predict differences in intelligence. Nat Commun 2018; 9: 1905.

36. Hibar DP, Westlye LT, Doan NT, et al. Cortical abnormalities in bipolar disorder: an 
MRI analysis of 6503 individuals from the ENIGMA Bipolar Disorder Working Group. Mol Psychiatry 2018; 23: 932-942.

37. Caverzasi E, Mandelli ML, Hoeft F, et al. Abnormal age-related cortical folding and neurite morphology in children with developmental dyslexia. Neuroimage Clin 2018; 18: 814-821.

38. Bracht T, Steinau S, Federspiel A, Schneider C, Wiest R, Walther S. Physical activity is associated with left corticospinal tract microstructure in bipolar depression. Neuroimage Clin 2018; 20: 939-945.

39. Czéh B, Nagy SA. Clinical Findings Documenting Cellular and Molecular Abnormalities of Glia in Depressive Disorders. Front Mol Neurosci 2018; 11: 56.

40. Tha KK, Terae S, Nakagawa S, et al. Impaired integrity of the brain parenchyma in non-geriatric patients with major depressive disorder revealed by diffusion tensor imaging. Psychiatry Res 2013; 212: 208-215. 


\section{TABLES}

Table 1. A summary of the demographic and clinical variables of the participants.

\begin{tabular}{lllll}
\hline & $\mathrm{BD}(\mathrm{n}=16)$ & $\mathrm{MDD}(\mathrm{n}=19)$ & Healthy volunteers $(\mathrm{n}=20)$ & Statistics \\
\hline Age (years) & $47.56(11.99)$ & $44.47(14.46)$ & $41.40(8.73)$ & $\mathrm{F}=1.20, \mathrm{P}=0.311$ \\
Gender (men/women) & $11 / 5$ & $8 / 11$ & $8 / 12$ & $\chi^{2}=3.51, \mathrm{P}=0.173$ \\
Total education (years) & $14.12(2.65)$ & $13.94(2.74)$ & $14.85(2.05)$ & $\mathrm{F}=0.717, \mathrm{P}=0.493$ \\
Total disease duration (months) & $256.81(160.91)$ & $163.39(124.30)$ & $\mathrm{t}=1.91, \mathrm{P}=0.066$ \\
Duration of the current disease & $59.88(26.40)$ & $261.11(250.19)$ & $\mathrm{t}=-3.20, \mathrm{P}=0.003$ \\
episode (days) & & & $\mathrm{t}=4.70, \mathrm{P}<0.001$ \\
Number of past manic/depressive & $8.38(5.49)$ & $2.11(1.32)$ & - & $\mathrm{t}=1.73, \mathrm{P}=0.092$ \\
episodes & & & $\mathrm{t}=-1.30, \mathrm{P}=0.204$ \\
YMRS & $1.44(2.37)$ & $0.42(0.90)$ & - & $\mathrm{t}=-1.80, \mathrm{P}=0.080$ \\
17-HDRS & $14.75(5.51)$ & $17.21(5.67)$ & - & - \\
MADRS & $18.37(8.69)$ & $24.36(10.62)$ & &
\end{tabular}




$\begin{array}{lcc}\text { complete remission } & 1 & 2 \\ \text { manic episode } & 0 & 0 \\ \text { depressive episode } & 14 & 17 \\ \text { mixed episode } & 1 & 0\end{array}$

$$
\chi^{2}=0.20, P=0.653
$$

Severity of depressive episode

mild depression

11

2

moderate depression

severe depression

1

$$
\begin{aligned}
& \chi^{2}=3.54, P=0.06 \\
& \chi^{2}=1.79, P=0.181 \\
& \chi^{2}=1.55, P=0.213
\end{aligned}
$$

Number of patients taking

$\begin{array}{ll}\text { antidepressant } & 7 \\ \text { mood stabilizer } & 14 \\ \text { antipsychotic drug } & 9\end{array}$

17

8

$$
\begin{aligned}
& \chi^{2}=8.43, \mathrm{P}=0.004 \\
& \chi^{2}=13.10, \mathrm{P}=0.001 \\
& \chi^{2}=0.70, \mathrm{P}=0.404
\end{aligned}
$$

The mean values and standard deviation in parentheses are given for age, total education, disease duration, number of past manic/depressive episodes, and the clinical scales.

Abbreviations: $\mathrm{BD}=$ bipolar disorder, $\mathrm{MDD}=$ major depressive disorder, YMRS= Young Mania Rating Scale, 17-HDRS= 17-item Hamilton 
Depression Rating Scale, MADRS= Montgomery-Åsberg Depression Rating Scale 
Table 2. The results of receiver-operating characteristics (ROC) analysis.

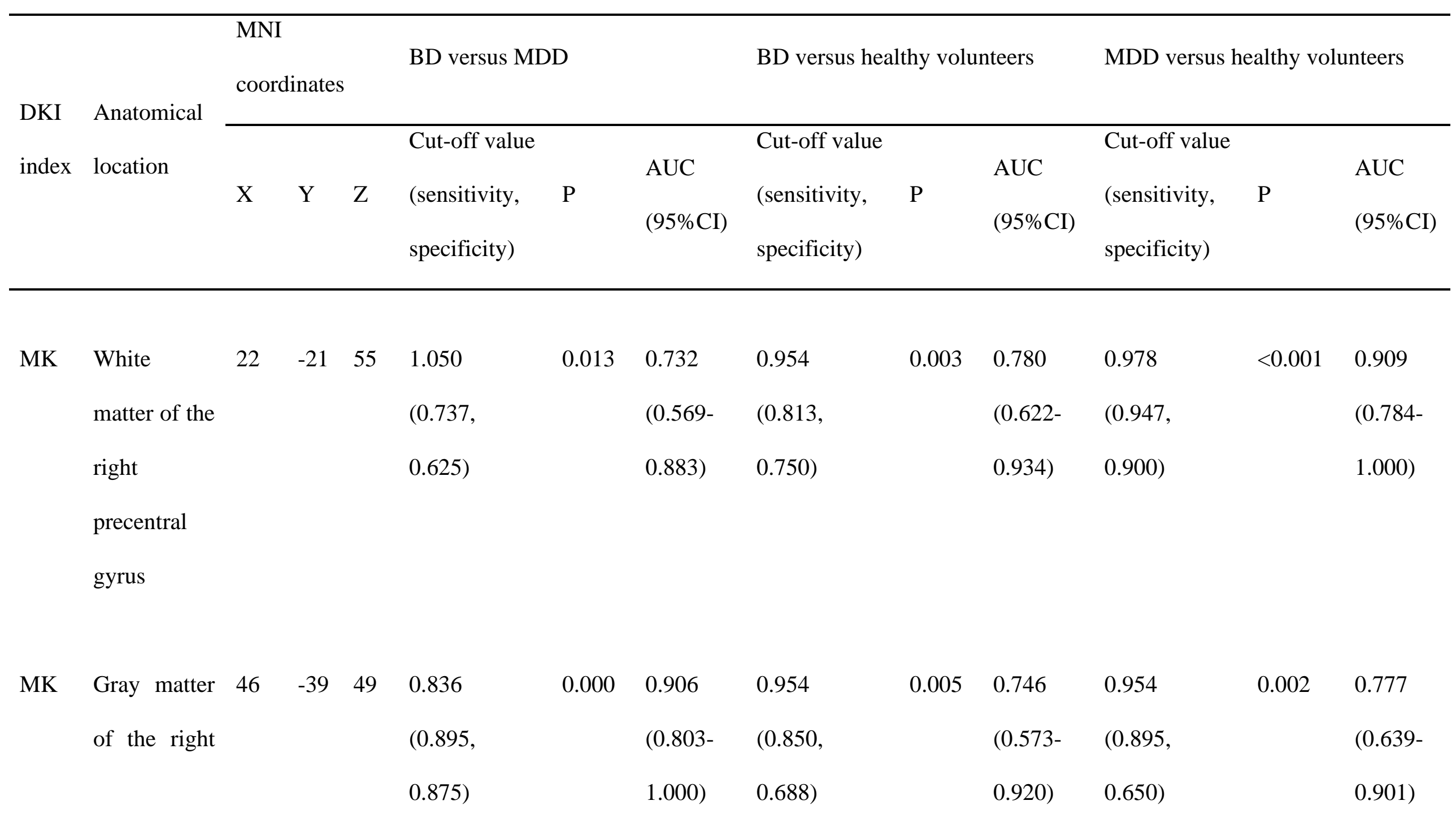


inferior

parietal lobe

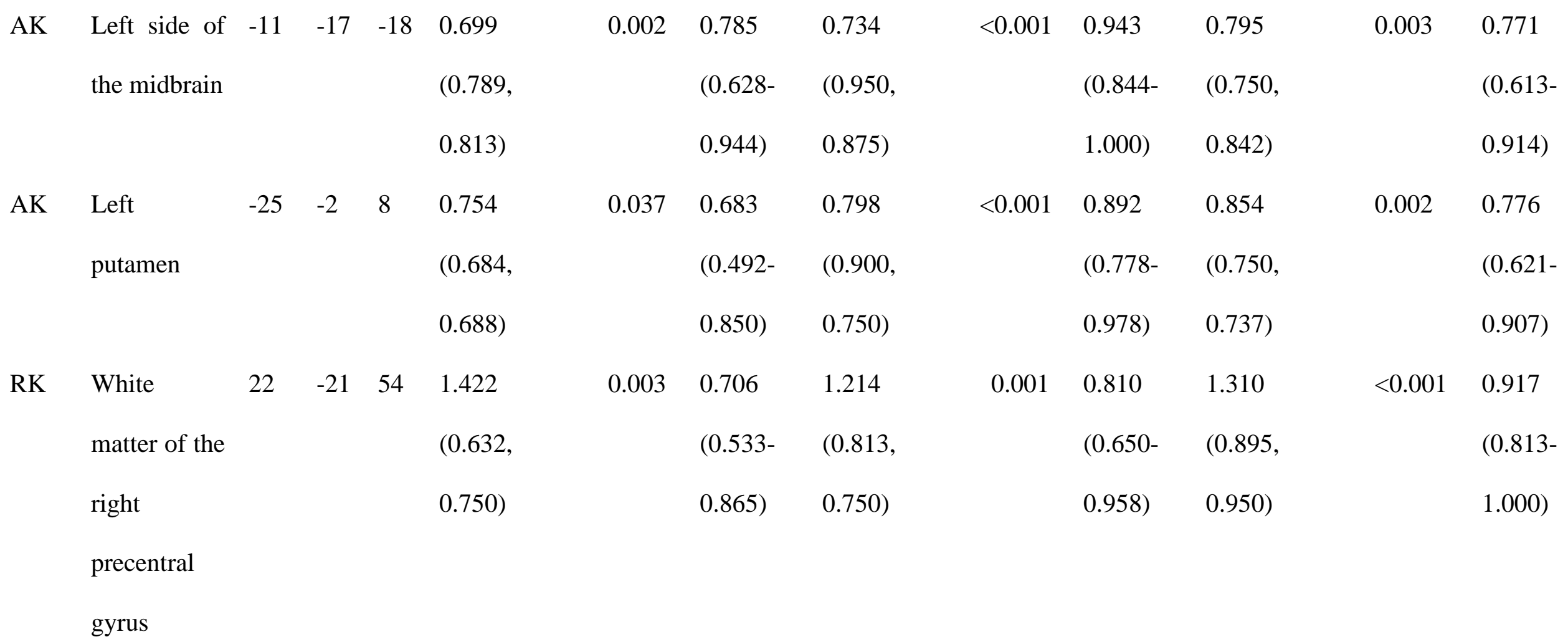


DKI Alterations in BD and MDD 26

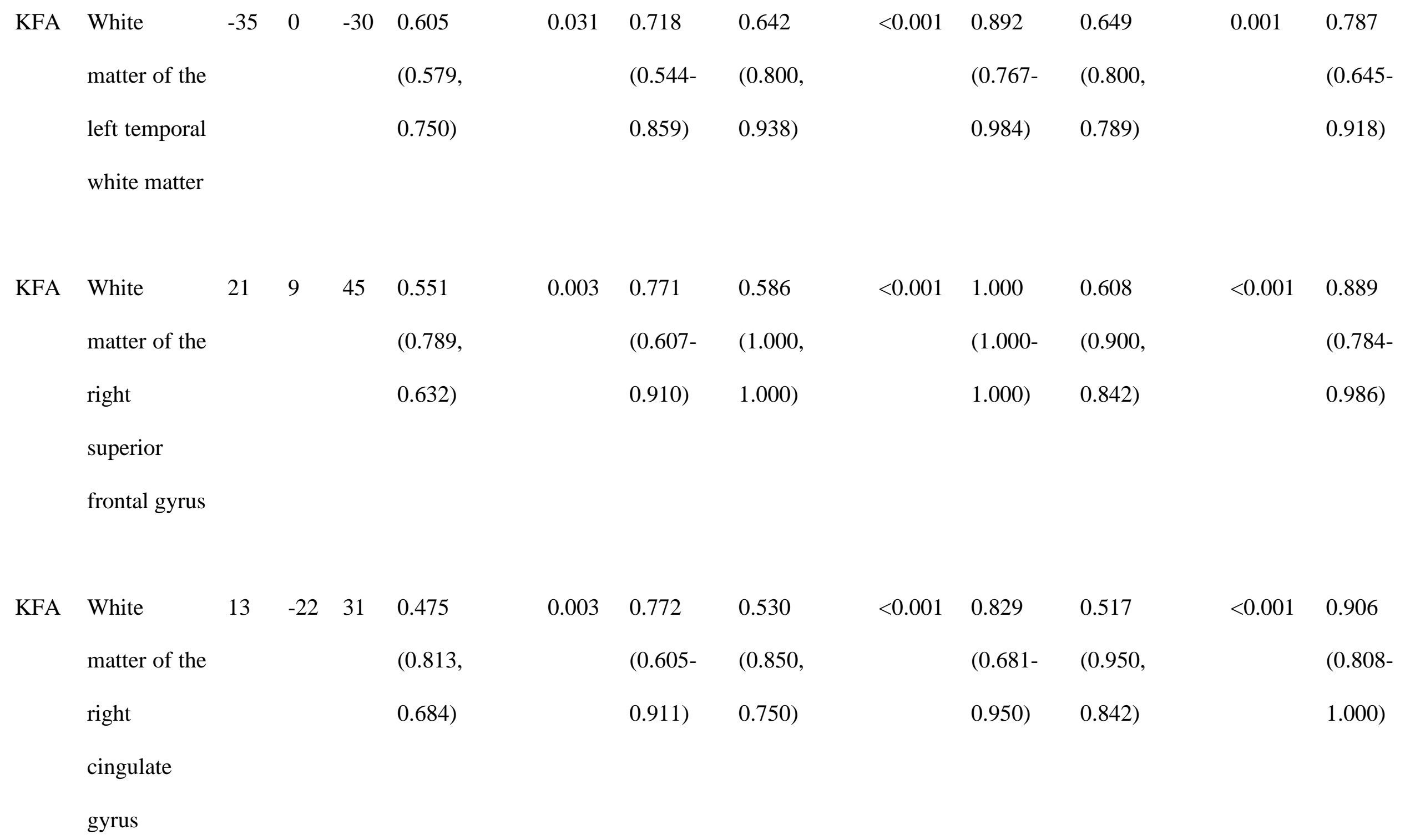




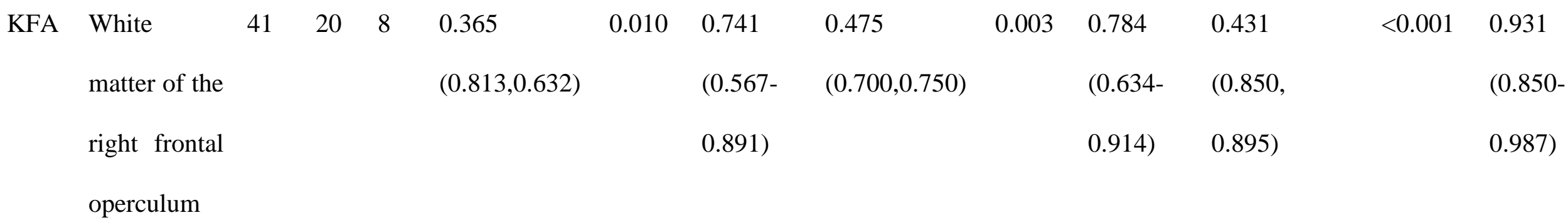

Sensitivity and specificity are given in \%.

Abbreviations: DKI= diffusion kurtosis imaging, MNI= Montreal Neurological Institute, BD= bipolar disorder, MDD= major depressive disorder, $\mathrm{AUC}=$ area under curve, $\mathrm{CI}=$ confidence interval, $\mathrm{MK}=$ mean kurtosis, $\mathrm{AK}=$ axial kurtosis, $\mathrm{RK}=$ radial kurtosis, $\mathrm{KFA}=$ kurtosis fractional anisotropy 


\section{FIGURE LEGENDS}

Fig 1. Clusters with significant difference (uncorrected $\mathrm{P}<0.001$, 50 voxels) in mean kurtosis (MK), axial kurtosis (AK), radial kurtosis (RK), mean diffusivity (MD), axial diffusivity (AD), radial diffusivity (RD), and kurtosis fractional anisotropy (KFA) between bipolar disorder (BD) and major depressive disorder (MDD), as analyzed by one-way analysis of variance (ANOVA) and Bonferroni post hoc tests. The clusters are overlaid on the axial magnetization-prepared rapid acquisition gradient-echo imaging (MPRAGE) images. The red, blue, and yellow colors in the uppermost row represent clusters with significantly higher MK, AK, and RK in MDD patients, respectively. The pink, cyan, and orange colors in the middle row represent clusters with significantly lower MD, AD, and RD in the MDD patients, respectively. The red and pink colors in the lowermost row represent clusters with significantly higher and lower KFA in the MDD patients. L indicates left.

Fig 2. The results of tests of correlation between mean kurtosis (MK) in the gray matter of the right inferior parietal lobe and (a) Young Mania Rating Scale (YMRS) in the bipolar disorder (BD) patients and (b) 17-item Hamilton Depression Rating Scale (17-HDRS) in the major depressive disorder (MDD) patients. The straight lines indicate the mean and the curved lines 95\% confidence interval. The black and gray circles indicate the patients in complete remission and the mixed episode, respectively.

Fig 3. The results of tests of correlation of the (a) kurtosis fractional anisotropy (KFA) of the right inferior frontal operculum of the major depressive disorder (MDD) patients, (b) mean diffusivity (MD) in the white matter of right orbital gyrus of the MDD patients, and (c) MD in the white matter of the left orbital gyrus of the bipolar disorder (BD) patients, with total disease duration or duration of current disease episode. The straight lines indicate the mean and the curved lines 95\% confidence interval. The black and gray circles indicate the patients in complete remission and the mixed episode, respectively. 


\section{FIGURES}

Fig 1

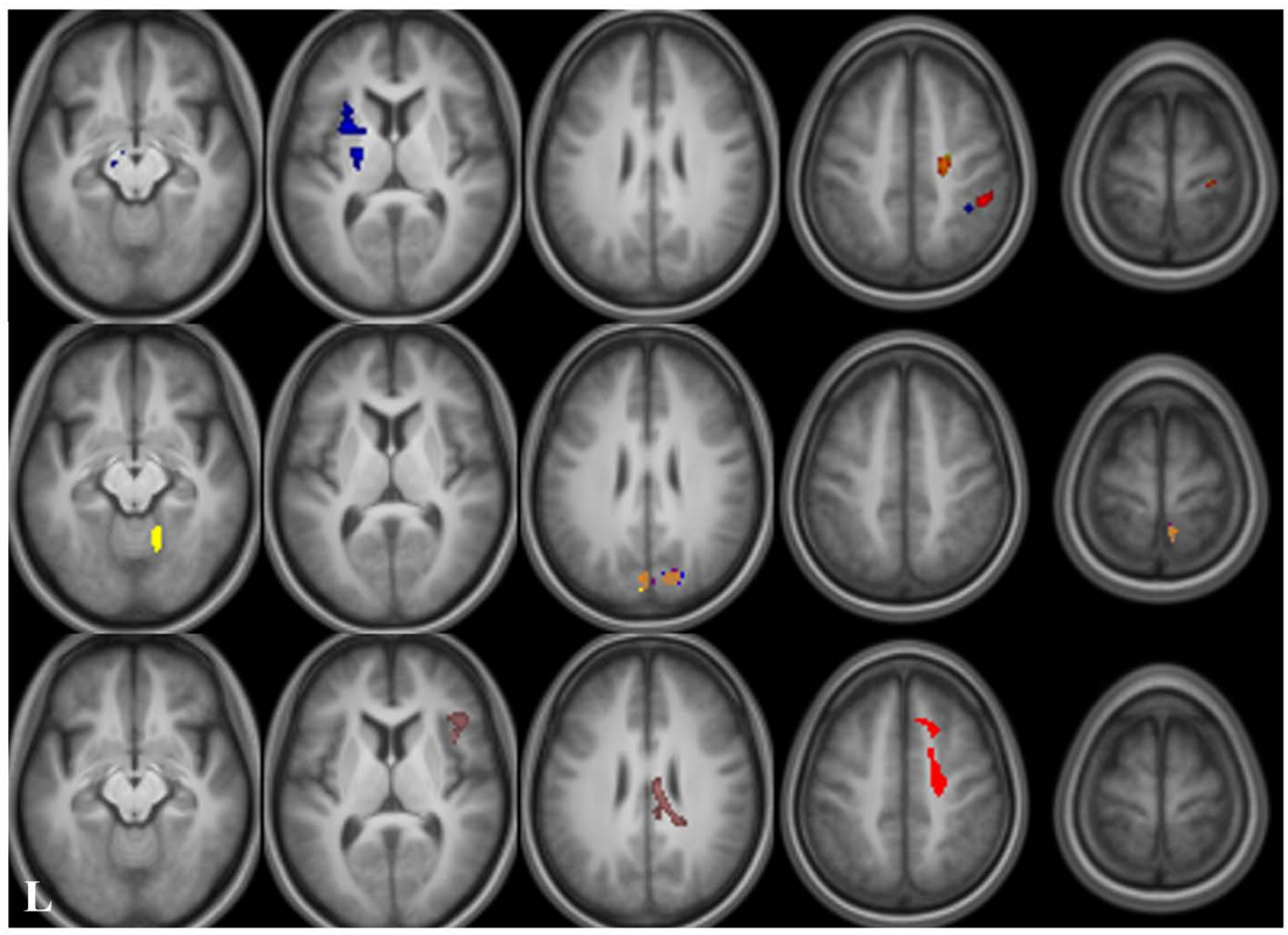


Fig 2a

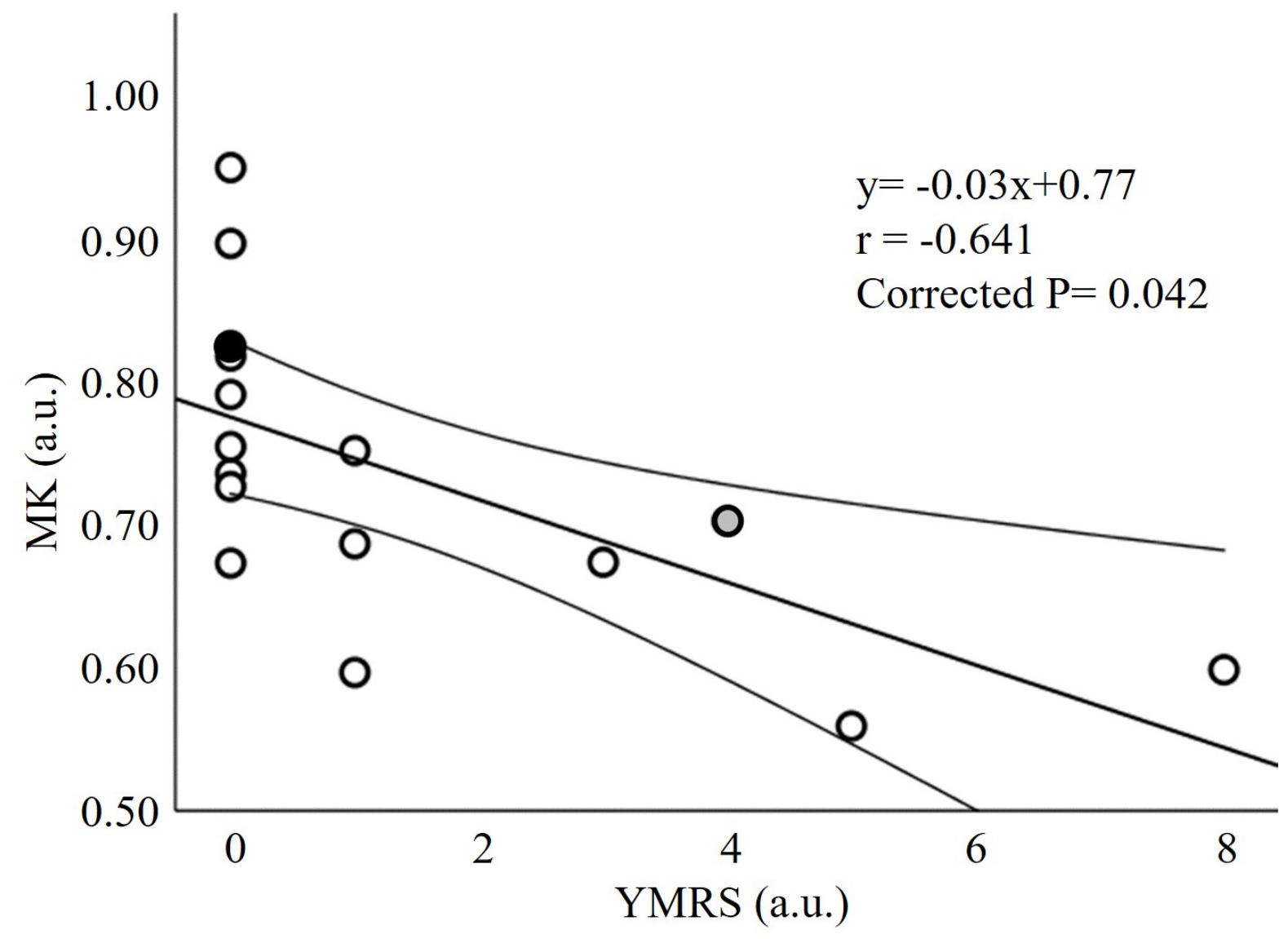


Fig 2b

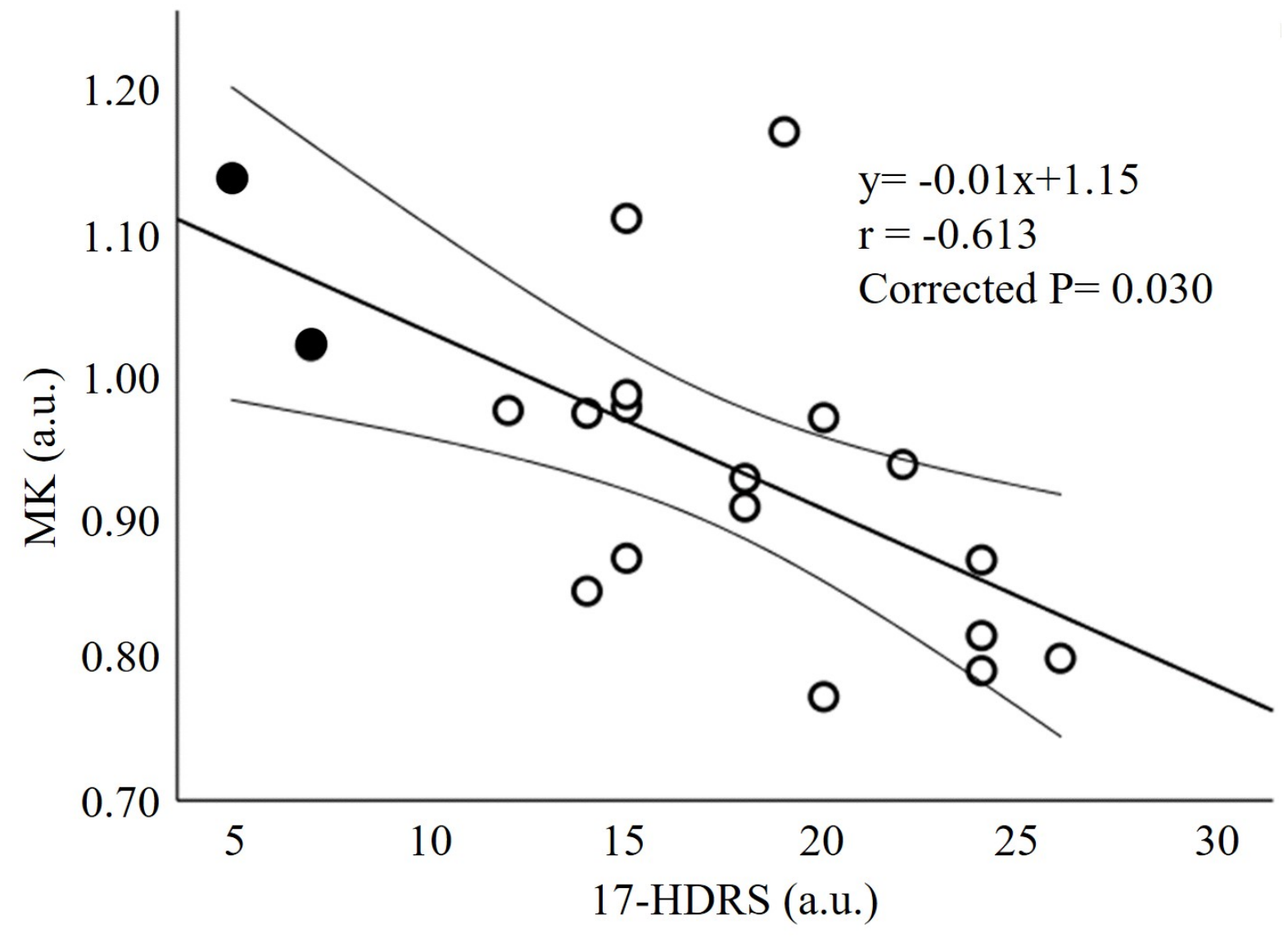


Fig 3a

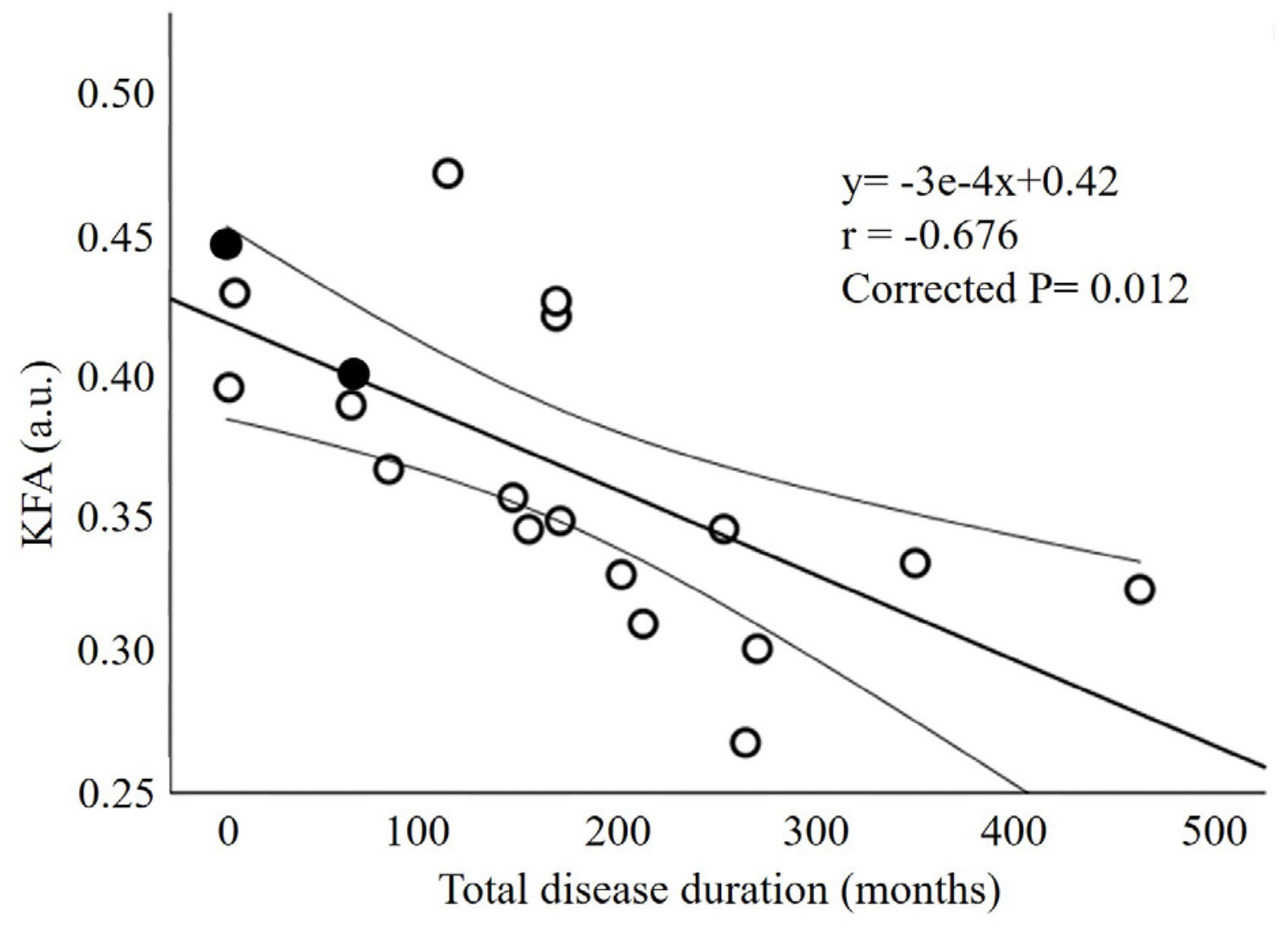


Fig 3b

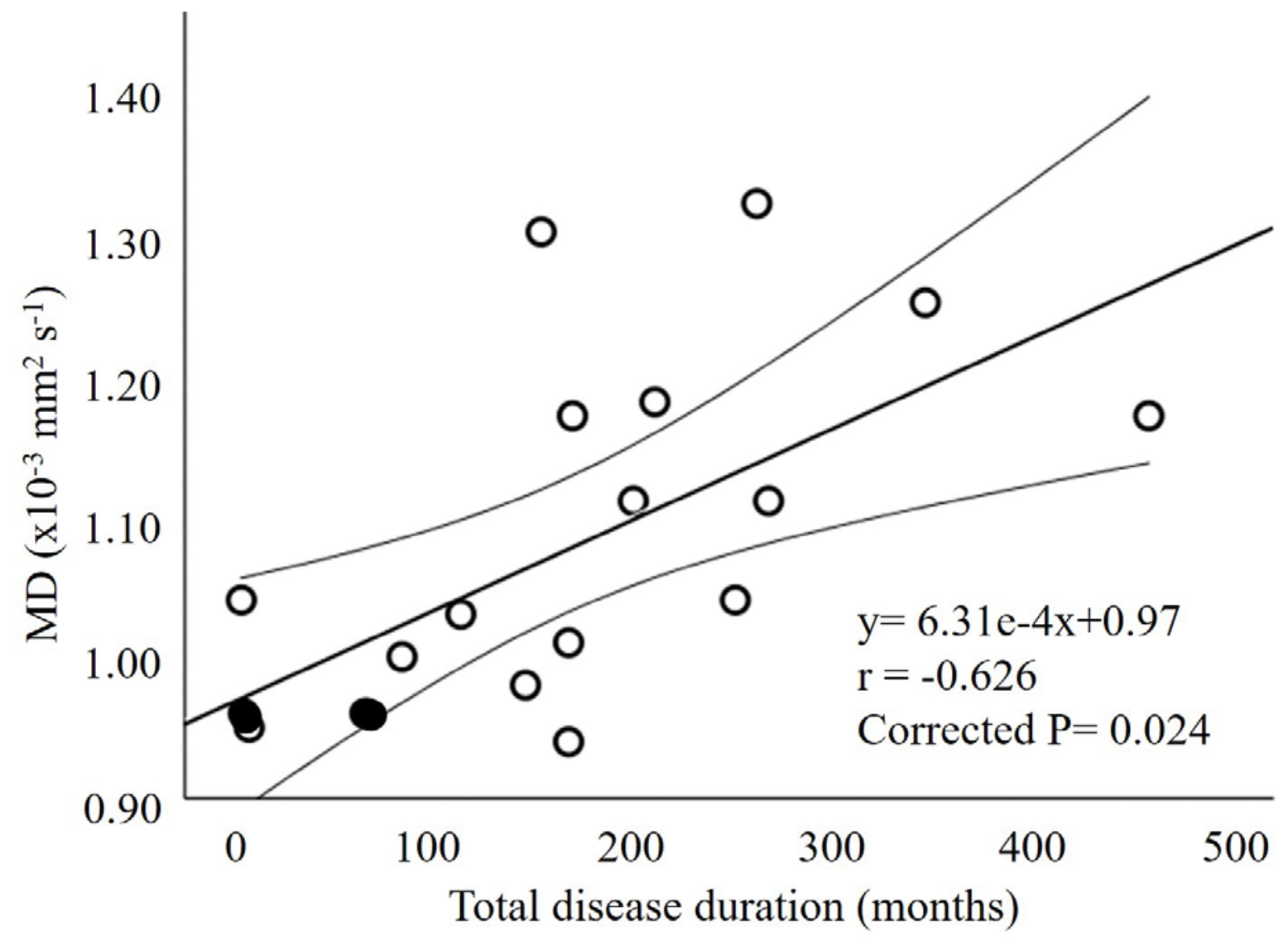


Fig 3c

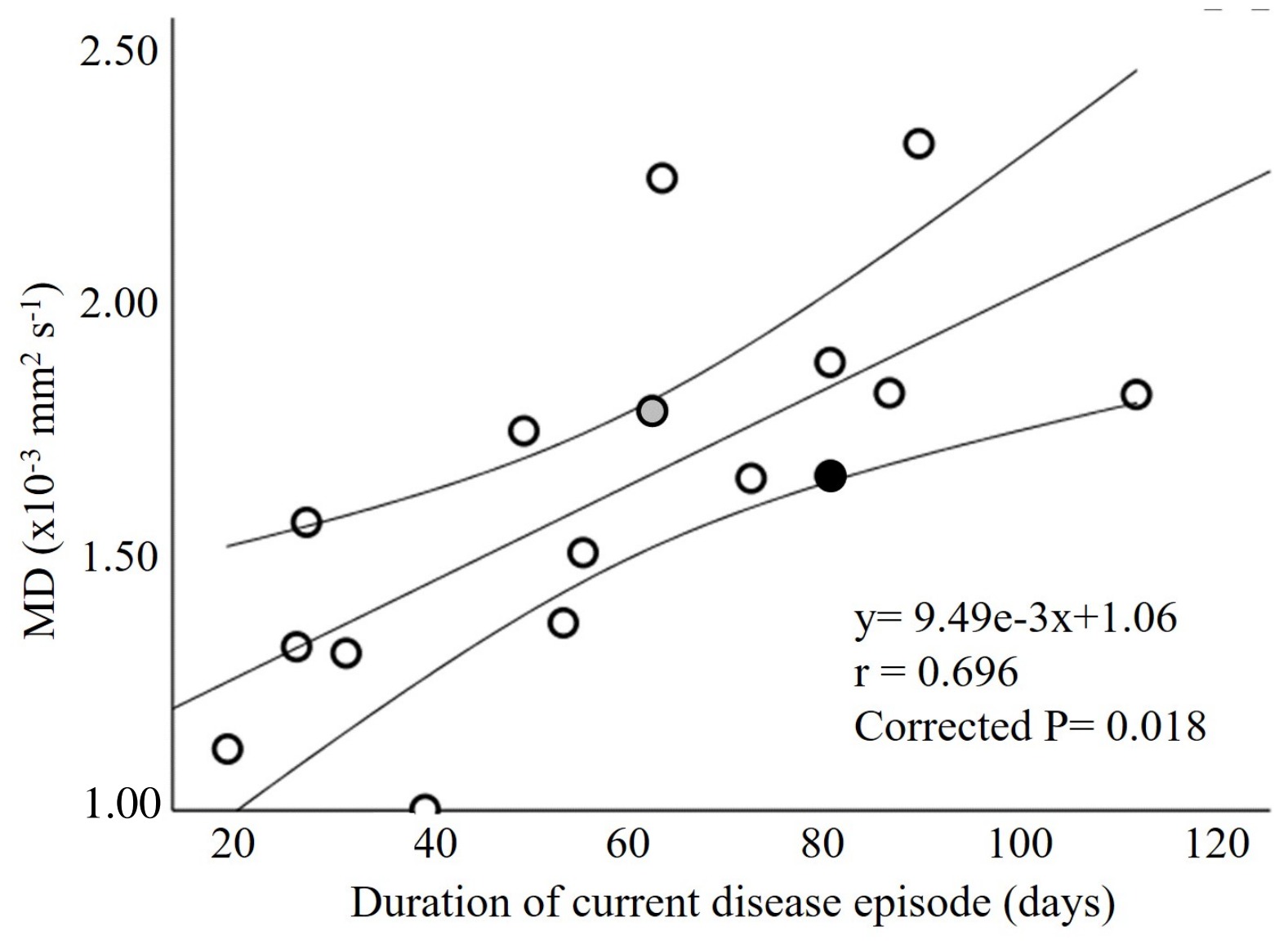




\section{SUPPLEMENTARY MATERIALS}

Fig 1s. The results of the voxel-by-voxel comparison of the diffusion kurtosis imaging (DKI) indices among the three groups, as analyzed by using one-way analysis of variance (ANOVA). Clusters showing significant differences in the DKI indices are shown (uncorrected $\mathrm{P}<0.001$, $\geq 50$ voxels), by overlaying on the axial MPRAGE images. Alterations in mean kurtosis (MK) (red), axial kurtosis (AK) (blue), and radial kurtosis (RK) (yellow) are shown in the uppermost row. Those in mean diffusivity (MD) (red), axial diffusivity (AD) (blue), and radial diffusivity (RD) (yellow) are shown in the middle row. Those in kurtosis fractional anisotropy (KFA) (red) and fractional anisotropy (FA) (blue) are shown in the lowermost row. L indicates left.

Fig 2s. The mean values of clusters which differ significantly between the patients receiving antipsychotic medications (a-e for antidepressant, f-h for mood stabilizer, or i-l for antipsychotic drug) and those who do not. * indicates $\mathrm{P}<0.05$. + denotes those receiving antipsychotic medication and - those who do not.

Table 1s. Clusters with significant difference in the DKI indices among the three groups. 